\title{
TU/e EmonONEN

\section{Scaling of the density of state of the weighted Laplacian in the presence of fractal boundaries}

\section{Citation for published version (APA):}

Adrover, A., \& Garofalo, F. (2010). Scaling of the density of state of the weighted Laplacian in the presence of fractal boundaries. Physical Review E - Statistical, Nonlinear, and Soft Matter Physics, 81(2), 027202-1/4. [027202]. https://doi.org/10.1103/PhysRevE.81.027202

DOI:

10.1103/PhysRevE.81.027202

Document status and date:

Published: 01/01/2010

\section{Document Version:}

Publisher's PDF, also known as Version of Record (includes final page, issue and volume numbers)

\section{Please check the document version of this publication:}

- A submitted manuscript is the version of the article upon submission and before peer-review. There can be important differences between the submitted version and the official published version of record. People interested in the research are advised to contact the author for the final version of the publication, or visit the $\mathrm{DOI}$ to the publisher's website.

- The final author version and the galley proof are versions of the publication after peer review.

- The final published version features the final layout of the paper including the volume, issue and page numbers.

Link to publication

\section{General rights}

Copyright and moral rights for the publications made accessible in the public portal are retained by the authors and/or other copyright owners and it is a condition of accessing publications that users recognise and abide by the legal requirements associated with these rights.

- Users may download and print one copy of any publication from the public portal for the purpose of private study or research.

- You may not further distribute the material or use it for any profit-making activity or commercial gain

- You may freely distribute the URL identifying the publication in the public portal.

If the publication is distributed under the terms of Article 25fa of the Dutch Copyright Act, indicated by the "Taverne" license above, please follow below link for the End User Agreement:

www.tue.nl/taverne

Take down policy

If you believe that this document breaches copyright please contact us at:

openaccess@tue.nl

providing details and we will investigate your claim. 


\title{
Scaling of the density of state of the weighted Laplacian in the presence of fractal boundaries
}

\author{
Alessandra Adrover* and Fabio Garofalo ${ }^{\dagger}$ \\ Dipartimento di Ingegneria Chimica, Materiali, Ambiente La Sapienza Università di Roma, Via Eudossiana 18, 00184 Roma, Italy
}

(Received 19 October 2009; published 19 February 2010)

\begin{abstract}
Spectral properties of the weighted Laplace operator in the presence of fractal boundaries are numerically investigated for both Neumann and Dirichlet boundary conditions. This corresponds to the characterization of heat and mass transport in microchannels with irregular and rough surfaces induced by the microfabrication process. The axial velocity field with no-slip boundary conditions, representing the weighting function of the Laplace operator, influences the localization properties of the eigenfunctions and the scaling of the integrated density of state (IDOS) $N(\lambda)$. The results indicate that $N(\lambda)$ deviates from the form given by the modified Weyl-Berry-Lapidus conjecture as it shows a correction of $\Delta N(\lambda) \sim \lambda^{D_{f} / 4}$ to the leading-order Weil term. Numerical results are presented for Koch and Koch snowflake fractal boundaries. The role of slip or no-slip boundary conditions of the velocity field on the IDOS is also investigated.
\end{abstract}

DOI: 10.1103/PhysRevE.81.027202

Macroscopic transport processes in the presence of irregular interfaces appear in many physical, geological, chemical, and biological systems [1]. Irregular and hierarchical structures can be described in terms of fractal concepts $[2,3]$.

Vibrational properties of surface fractal resonators (fractal drums) have been largely investigated theoretically [4-6], numerically $[7,8]$, and experimentally $[9,10]$ by analyzing vibrational spectra, localization mechanisms, the structure of their vibrational modes, and the damping of these modes.

From a mathematical point of view this corresponds to the study of the eigenvalue equation of the Laplace operator $-\lambda \psi=\nabla^{2} \psi$ and of the asymptotic properties of the integrated density of states (IDOS) $N(\lambda)$ in the presence of fractal boundary $[4,5]$.

The results show that the low-frequency IDOS is well approximated by a two term expression given by the WeylBerry-Lapidus (WBL) conjecture [4] which predicts a correction of $\Delta N(\lambda) \sim \lambda^{D_{f} / 2}$ to the leading-order term

$$
N(\lambda)=(S / 4 \pi)\left(\lambda+\Delta N(\lambda)=(S / 4 \pi) \lambda-B_{f} \lambda^{D_{f}^{\prime 2}},\right.
$$

where $D_{f}$ is the fractal dimension of the perimeter, $S$ is the area of the drum, and $B_{f}$ is a positive constant depending on the shape of the drum. In the high-frequency regime [7,8], where the half wavelength is smaller than the smallest feature of the fractal (prefractal) perimeter, the two term Weyl asymptotic is applicable with $\Delta N(\lambda) \sim \lambda^{1 / 2}$.

However, the dynamics of several physical, biophysical, and geophysical phenomena is governed by the interplay between advection and diffusion in evolving scalar and vector fields [11-13]. Advection-diffusion dynamics in laminar flows has been extensively studied in closed systems (see [14-16] and reference therein), but much less is known on the fundamental physical processes characterizing convective-diffusive transport in open flows even though continuous flow devices represent the most common process units in classical (e.g., polymer processing in static mixers and extruders [17]), and advanced applications (microfludic systems, see, e.g., $[18,19])$.

\footnotetext{
*alessandra.adrover@uniroma1.it

†fabio.garofalo@uniroma1.it
}

PACS number(s): 05.45.Df, 47.53.+n, 63.50.- $-\mathrm{x}$

When transport Laplacian-based processes occurs in the presence of flow fields, such as solute dispersion or heat transport in microchannels, system analysis, and characterization requires the investigation of a more general problem (with respect to that of fractal drums), i.e., the spectral characterization of the velocity-field-weighted Laplacian in the presence of both Dirichlet (for thermal problems) or Neumann boundary conditions (for mass transport problems). Moreover, in microfluidic systems, boundary effects are important because of the large surface to volume ratio and surface roughness, e.g., induced by the microfabrication process [20], may play a fundamental role on transport processes [21].

Let us consider laminar fluid flow in a microchannel of length $L$ and rectangular cross section $\Omega$ of characteristic length $W$, with regular or fractal boundary $\partial \Omega$. By neglecting the contribution of axial dispersion neglecting axial dispersion (NAD approximation [22]) the spatial evolution of a steady-state scalar field $\phi\left(x_{\perp}\right)$ within the channel can be modeled via the advection-diffusion equation

$$
v_{z}\left(x_{\perp}\right)(\partial \phi / \partial z)=\left(\alpha^{2} / \mathrm{Pe}\right) \nabla_{\perp}^{2} \phi,
$$

where $\alpha$ is the channel aspect ratio $L / W, \mathrm{Pe}=V_{\text {ref }} L / \mathcal{D}$ $>10 \alpha^{2}$ is the axial Peclet number and $v_{z}\left(x_{\perp}\right)$ is the Stokes velocity field, solution of the two-dimensional Poisson problem

$$
\nabla^{2} v_{z}\left(x_{\perp}\right)=-A,\left.\quad v_{z}\right|_{\partial \Omega}=0,
$$

$A$ being a normalizing constant that yields, e.g., unitary flow rate.

For a solute dispersion experiment as well as for the heat transport problem, all transport information is embedded in the spectral properties of the weighted Laplace operator $\mathcal{L}_{v}(\psi)=\left[1 / v_{z}\left(x_{\perp}\right)\right] \nabla_{\perp}^{2} \psi, \quad$ i.e., within the eigenvalue/ eigenfunction spectrum associated with the eigenvalue equation

$$
-\lambda v_{z}\left(x_{\perp}\right) \psi=\nabla_{\perp}^{2} \psi
$$

equipped with Dirichlet $\left.\psi\right|_{\partial \Omega}=0$ or Neumann $\partial \psi / \partial n=\left.0\right|_{\partial \Omega}$ boundary conditions for heat and mass transport problem, respectively. 


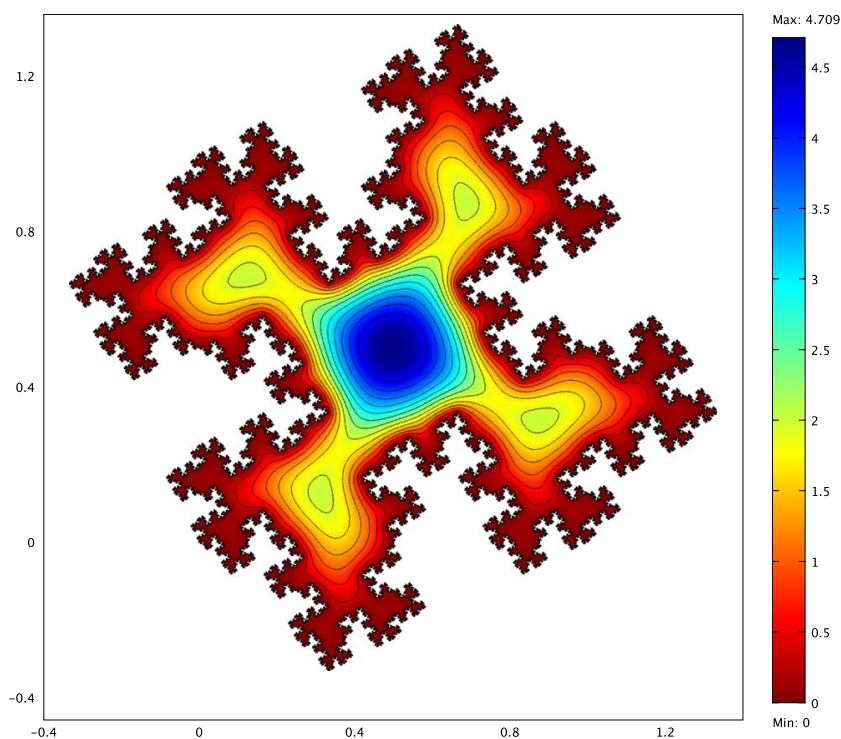

(a)

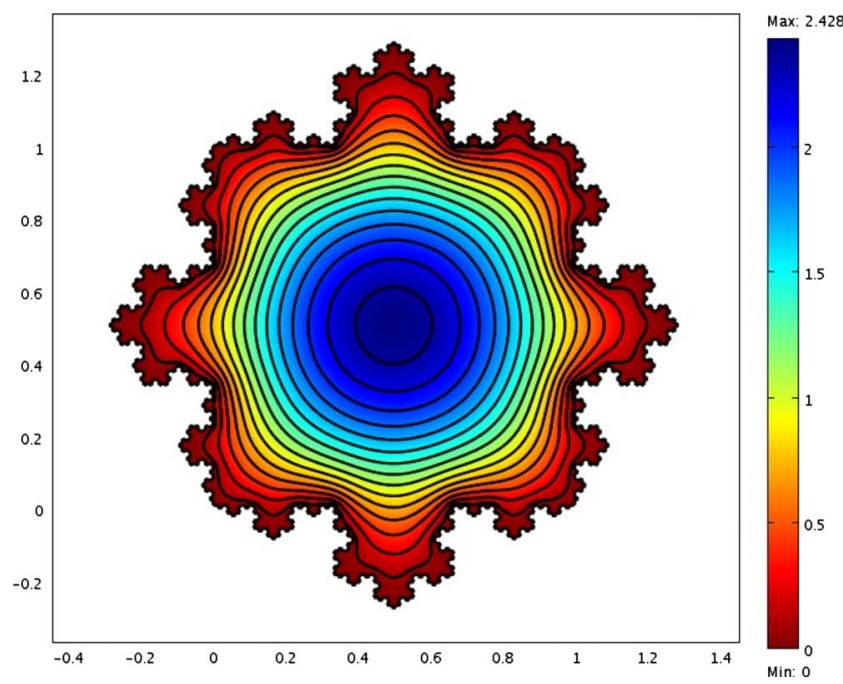

(b)

FIG. 1. (Color online) Contour color-plot of the velocity field $v_{z}\left(x_{\perp}\right)$ with no-slip boundary conditions for the two fractal microchannels considered. (a) Koch structure $(\nu=4)$; (b) Koch snowflake $(\nu=5)$.

We consider two microchannels with fractal boundaries: a Koch structure of fractal dimension $D_{f}=3 / 2$ at prefractal generations $\nu=3-4$ and the Koch snowflake $\left[D_{f}\right.$ $=\ln (4) / \ln (3)]$ at generations $\nu=4-5$. Figures $1(\mathrm{~A})$ and $1(\mathrm{~B})$ show the velocity fields $v_{z}\left(x_{\perp}\right)$ with no-slip boundary conditions for the two microchannels at the highest prefractal iteration considered.

We show that the no-slip boundary conditions for the weighting function velocity field modify significantly the scaling properties of the integrated density of state and prevent the localization of the wave amplitude near the fractal boundary.

Figures 2(A) and 2(B) show the behavior of the IDOS $N(\lambda)$ and of the subleading-order term $\Delta N(\lambda)$ for the Koch structure and the Koch snowflake at the highest prefractal generation considered. It can be observed that the leading-
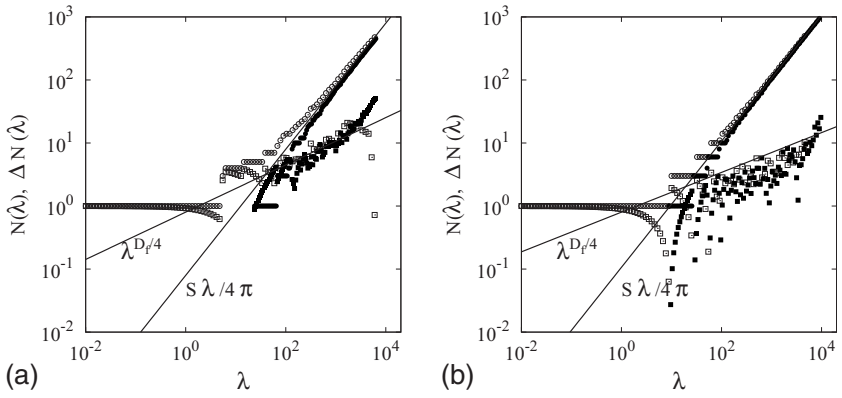

FIG. 2. IDOS $N(\lambda)$ (open and closed circles) and subleadingorder term $\Delta N(\lambda)$ (open and closed squares) for the two systems considered at the highest generation order analyzed. (a) Koch structure $(\nu=4)$; (b) Koch snowflake $(\nu=5)$. Open and closed points correspond to homogeneous Neumann and Dirichlet boundary conditions, respectively. Continuous lines show the conjectured scaling of the leading-order term $S \lambda / 4 \pi$ and of the subleading-order term $\Delta N(\lambda) \sim \lambda^{D_{f} / 4}$.

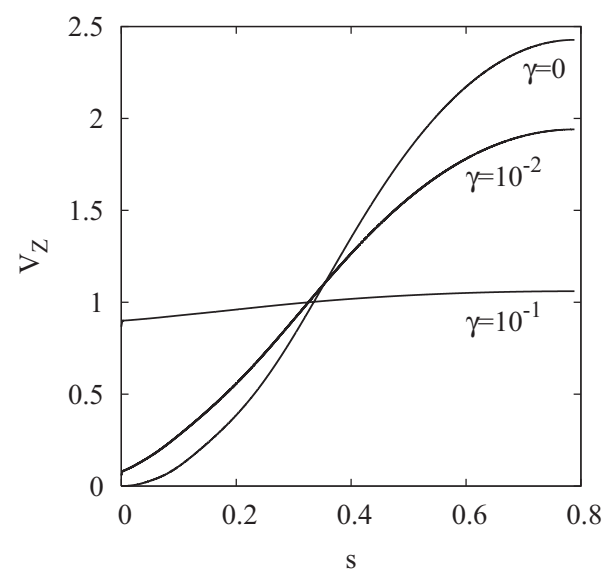

FIG. 3. Axial velocity field $v_{z}$ as a function of the vertical distance from the highest point of the Kock snowflake for no-slip $(\gamma$ $=0)$ and slip boundary conditions with $\gamma=10^{-2}$ and $\gamma=10^{-1}$.

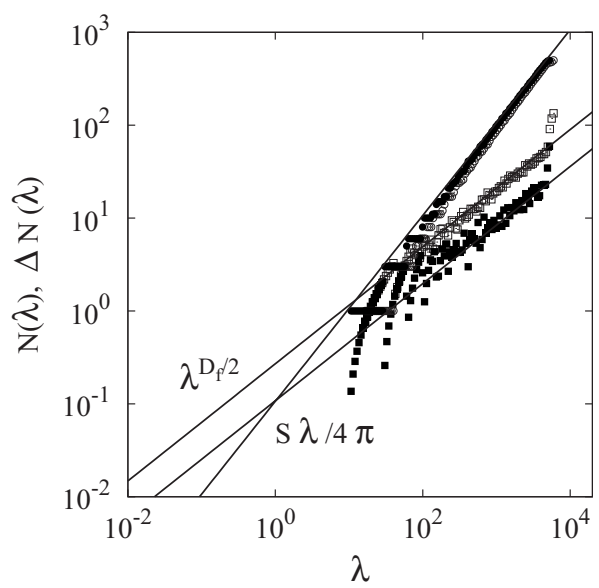

FIG. 4. IDOS $N(\lambda)$ (open and closed circles) and subleadingorder term $\Delta N(\lambda)$ (open and closed squares) for the Koch snowflake $(\nu=5)$ in the presence of a velocity field satisfying slip boundary conditions for two different values of the slippage coefficient $\gamma ; \gamma$ $=10^{-1}$ open points; $\gamma=10^{-2}$ closed points. Only homogeneous Dirichlet boundary conditions in the spectral analysis are considered. Continuous lines show the expected scaling of the leading-order term $S \lambda / 4 \pi$ and of the subleading-order term $\Delta N(\lambda) \sim \lambda^{D_{f} / 2}$. 


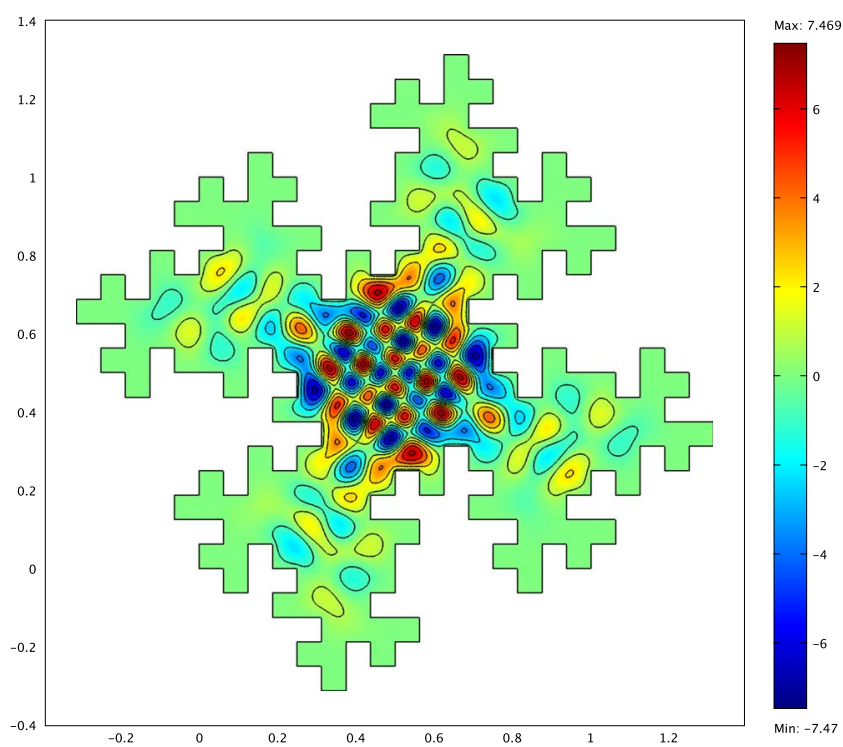

(a)

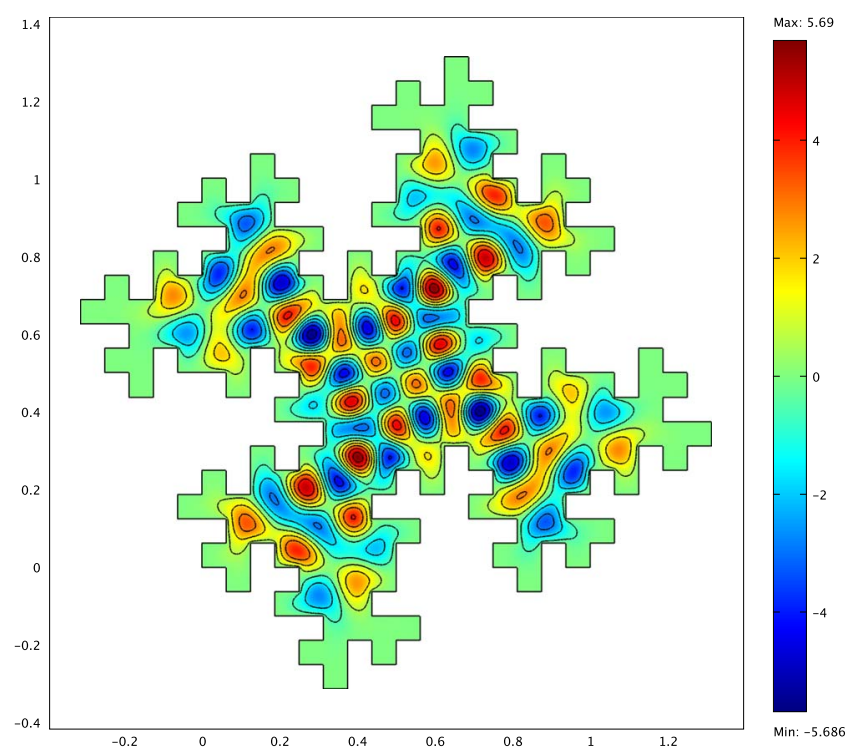

(b)

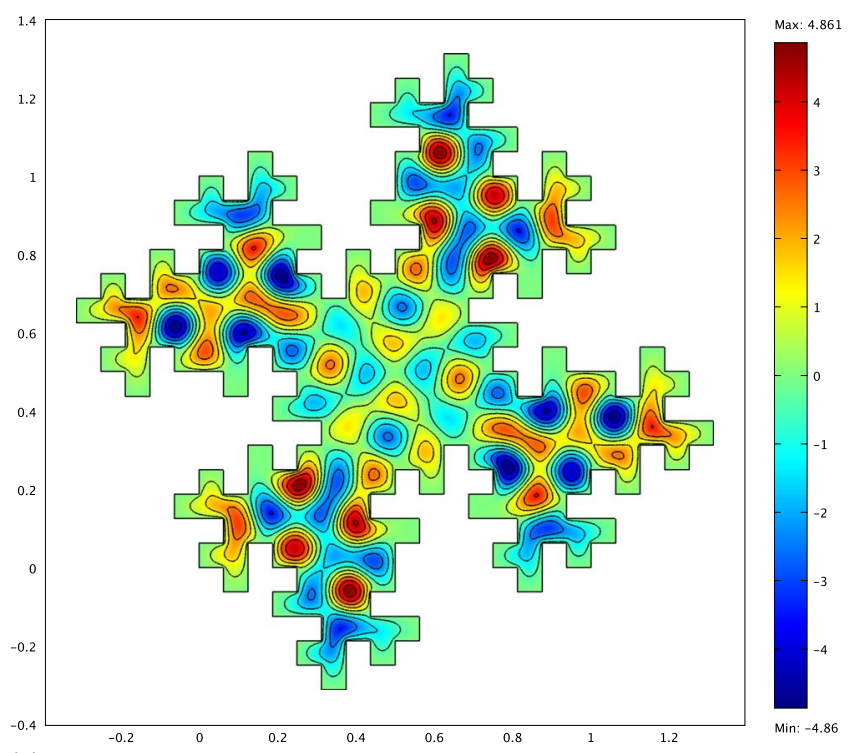

(c)

FIG. 5. (Color online) Eigenfunctions of the weighted Laplacian with Dirichlet boundary conditions for the Koch structure at the second prefractal generation $\nu=2$. (a) No-slip boundary conditions, $\lambda=1774$. (b) Slip boundary conditions $\gamma=10^{-2}$ and $\lambda=1755$. (c) Uniform and constant weighting function, $\lambda=1756$.

order term is unaffected by the weighting function, according with the result presented in [23] for the weighted $p$-Laplacian in one dimension. Yet, the velocity field plays a significant role, as it influences the scaling of the subleadingorder term so that the following general form can be conjectured

$$
N(\lambda)=(S / 4 \pi) \lambda \mp B_{f} \lambda^{D_{f} / 4},
$$

where the sign (-) and (+) apply for the Dirichlet (closed points in Fig. 2) and Neumann (open points in Fig. 2), respectively. Similar results, not shown here for the sake of brevity, are obtained for lower prefractal generation levels.

The spectrum has been obtained by means of a Krilov method applied to a finite element representation of the weighted Laplacian (from $5 \times 10^{5}$ to $2 \times 10^{6}$ triangular elements)

Deviation from the WBL conjecture [we observe $\Delta N(\lambda)$ $\sim \lambda^{D_{f} / 4}$ instead of $\left.\Delta N(\lambda) \sim \lambda^{D_{f} / 2}\right]$ are intrinsically due to the vanishing value of the weighting function at the fractal boundary induced by the no-slip boundary conditions for the Stokes flow. This can be numerically checked by analyzing the behavior of the IDOS for a weighting function associated with a velocity field with slip boundary conditions at the fractal boundary, i.e.,

$$
\left.\gamma\left(\partial v_{z} / \partial n\right)\right|_{\partial \Omega}=-\left.v_{z}\right|_{\partial \Omega} .
$$

Figure 3 shows the behavior of the velocity field $v_{z}$ as a function of the vertical distance $s$ from the highest point of 
the Koch snowflake structure for the no-slip $(\gamma=0)$ and slip boundary conditions with $\gamma=10^{-2}$ and $\gamma=10^{-1}$, corresponding to increasing values of the velocity at the fractal boundary.

Figure 4 shows the behavior of $N(\lambda)$ and of the subleading-order term $\Delta N(\lambda)$ for the Koch snowflake with slip boundary conditions for the velocity field and the two different values of $\gamma$ considered. In both cases we observe $N(\lambda) \sim \lambda^{D_{f} / 2}$ so that nor the leading neither the subleadingorder term deviate from the WBL scaling corresponding to a spatially uniform velocity field (constant weighting function).

A physical interpretation of the results presented is that if the IDOS is significantly decreased by the irregularity of the boundary [so that $\Delta N(\lambda) \sim \lambda^{D_{f} / 2}$ instead of $\Delta N(\lambda) \sim \lambda^{1 / 2}$ for a regular boundary], the effect of the no-slip weighting function (which therefore vanishes a the boundary) is to soften (attenuate) the influence of the fractal dimension on the IDOS [so that $\Delta N(\lambda) \sim \lambda^{D_{f} / 4}$. This effect is also due the presence of corner points, which are dense on the crosssectional perimeter. These points are critical in that the noslip condition implies that the velocity field scales quadratically as a function of the wall-distance $s$, and this effect is not present in the case of slip boundary conditions [where we observe $\Delta N(\lambda) \sim \lambda^{D_{f} / 2}$ ] where the velocity field scales linearly with $s$ also in correspondence of the corner points [24]. However, the origin of the scaling $\Delta N(\lambda) \sim \lambda^{D_{f} / 4}$ has not been well understood, and we emphasize the necessity of further analytical investigation for elucidating the numerical findings.

The vanishing value of the weighting function at the fractal boundary does influence the structure of the eigenfunctions and prevents the localization of the wave amplitude near the fractal boundary. This can be appreciated by comparing the eigenfunctions associated to similar eigenvalues in the case of no-slip [Fig. 5(A)], slip boundary conditions [Fig.
5(B) for $\left.\gamma=10^{-2}\right]$, and constant weighting function $v_{z}=1$ [corresponding to the classical fractal drum problem, Fig. $5(\mathrm{C})]$. For this qualitative comparison we consider the Koch fractal boundary at prefractal generation $\nu=2$ and eigenvalues or eigenfunctions corresponding to wavelengths of the order of the minimum characteristic length scale of the structure.

It can be observed that for no-slip boundary conditions, wave amplitude is actually localized in the mainland of the structure. When slip boundary conditions are considered, the eigenfunction amplitude exhibits significant oscillations close to the prefractal boundary and keeps on penetrating toward the dead ends of the prefractal perimeter when a constant weighting function is considered.

In summary, we have computed the IDOS of the weighted Laplacian in the presence of fractal boundaries. We have numerically shown that a weighting function, vanishing at the fractal or prefractal boundary influences the eigenfunction localization properties as well as the scaling properties of the IDOS as it shows a correction of $\Delta N(\lambda) \sim \lambda^{D_{f} / 4}$ to the leading-order Weil term.

The result presented in this Brief Report, representing the generalization of the fractal drum problem in the presence of variable mass density [25], may be relevant for microfluidic applications when one considers that modern microfabrication techniques can control superficial defects and roughness up to the nanolength scale. The systems considered possess the translational symmetry as they consist of a cross section with fractal boundary extruded along the longitudinal direction. Therefore the present analysis should be further generalized and complemented to investigate the effects of roughness and irregularities along the longitudinal direction. We note that this step (and the connection with the results presented in this Brief Report) is not altogether trivial as it requires us to consider a fully three-dimensional problem for both the velocity field and the eigenfunctions.
[1] B. Sapoval, in Fractals and Disordered Systems, edited by A. Bunde and S. Havlin (Springer-Verlag, Berlin, 1996).

[2] B. B. Mandelbrot, The Fractal Geometry of nature (W. H. Freeman, New York, 1983).

[3] J. Feder, Fractals (Plenum, New York, 1988).

[4] M. Lapidus, Trans. Am. Math. Soc. 325, 465 (1991).

[5] J. Kigami and M. L. Lapidus, Commun. Math. Phys. 158, 93 (1993).

[6] M. Lapidus and C. Pomerance, Math. Proc. Cambridge Philos. Soc. 119, 167 (1996).

[7] Y. Hobiki et al., Phys. Rev. E 52, R1310 (1995).

[8] S. Homolya et al., Phys. Rev. E 67, 026211 (2003).

[9] O. Haeberle et al., Appl. Phys. Lett. 73, 3357 (1998).

[10] C. Even et al., Phys. Rev. Lett. 83, 726 (1999).

[11] A. J. Majda and P. R. Kramer, Phys. Rep. 314, 237 (1999).

[12] I. Scheuring et al., Freshw. Biol. 45, 123 (2000).

[13] D. Perugini et al., J. Volcanol. Geotherm. Res. 124, 255 (2003).

[14] S. Cerbelli et al., Chem. Eng. Sci. 59, 2125 (2004).

[15] M. Giona et al., Phys. Rev. E 70, 046224 (2004).

[16] M. Giona et al., J. Fluid Mech. 513, 221 (2004).
[17] E. S. Szalai and F. Muzzio, AIChE J. 49, 2687 (2003).

[18] N.-T. Nguyen and S. T. Wereley, Fundamentals and Applications of Microfluidics (Artech House, Norwood, MA, 2006).

[19] T. M. Squires and S. R. Quake, Rev. Mod. Phys. 77, 977 (2005).

[20] W. Ehrfeld et al., Microreactors (Wiley-VCH, Weinheim, 2000).

[21] C. Kunert and J. Harting, Int. J. Comput. Fluid Dyn. 22, 475 (2008).

[22] S. Cerbelli et al., Microfluid. Nanofluid. 6, 747 (2009).

[23] J. F. Bonder and J. P. Pinasco, Ark. Mat. 41, 267 (2003).

[24] In order to verify the role of the no-slip weighting function in the non-fractal case we also computed the IDOS for the case of a regular square cross-section microchannel and analyzed the scaling behavior of the reminder term (results not reported here). This computation shows that, for this simpler structure, $\Delta N(\lambda) \sim \lambda^{1 / 4}$, in agreement with the result $\Delta N(\lambda) \sim \lambda^{D_{f} / 4}$ obtained for the two fractal structures.

[25] J. Fleckinger and M. L. Lapidus, Proc. Am. Math. Soc. 123, 513 (1995). 